\title{
Outcomes when Switching from a pro re nata Regimen to a Treat and Extend Regimen Using Aflibercept in Neovascular Age-Related Macular Degeneration
}

\author{
Helena Giannakaki-Zimmermann Andreas Ebneter Marion R. Munk \\ Sebastian Wolf Martin S. Zinkernagel \\ Department of Ophthalmology, Inselspital, Bern University Hospital, University of Bern, Bern, Switzerland
}

\author{
Keywords \\ Neovascular age-related macular degeneration · Intravitreal \\ injections · Aflibercept · Pro re nata regimen - Treat and \\ extend regimen
}

\begin{abstract}
Purpose: To investigate outcomes in patients with neovascular age-related macular degeneration (AMD) switched from a pro re nata regimen (PRN) to a treat and extend regimen (TER) under aflibercept. Procedure: Thirty-two patients were observed over 2 years: the first year on PRN and the second year on TER. Best-corrected visual acuity (BCVA) and central retinal thickness (CRT) were evaluated. Intra- and subretinal fluid as well as the number of visits and injections were assessed. Results: Both regimens resulted in a stable BCVA. Patients in TER had a significant decrease of CRT after 1 year compared to 1 year of treatment on PRN ( $p<0.0001)$. TER resulted in significantly less visits; however, significantly more injections were observed over the course of 1 year compared to PRN (10.25 vs. 7.5, $p<0.0001$ and 5.97 vs. 7.5, $p=0.0002$, respectively). Conclusion: A switch from PRN to TER in patients treated with aflibercept for AMD appears to be safe.

(c) 2016 S. Karger AG, Basel
\end{abstract}

\section{KARGER}

E-Mail karger@karger.com

www.karger.com/oph

\section{Introduction}

Antiangiogenic agents have revolutionized the treatment of wet age-related macular degeneration (AMD) in the last decades. However, the initial enthusiasm for this treatment has been dampened by the need of monthly injections to obtain good functional outcomes. To avoid the associated treatment burden for patients and related health care costs, alternative treatment regimens were developed to reduce the number of injections needed. Pro re nata (PRN) or treat as needed is one of the attempts to lower the number of injections while trying to obtain good functional outcomes in terms of best-corrected visual acuity (BCVA). Several studies, such as the CATT and the IVAN trials, showed that a PRN regimen required up to $50 \%$ fewer injections compared to a monthly regimen [1]; however, PRN was shown to lead to marginally inferior functional outcomes [2-4] and less reduction of central retinal thickness (CRT) compared to monthly injections [1]. One disadvantage for both, the PRN and for the monthly regimen is the need for monthly visits. A more recently introduced strategy to optimize the benefit:risk ratio is the treat and extend regimen (TER), which usually involves fixed treatment intervals until 
clinical remission is seen in optical coherence tomography (OCT), followed by either extension or decrease of treatment intervals, depending on OCT results $[5,6]$. Although there is a relative paucity of data on TER therapy, it has been adopted by the majority of AMD-treating physicians, mainly because it decreases the frequency of office visits while minimizing disease activity [1]. A recent study compared ranibizumab to bevacizumab using a treat and extend protocol and found a comparable visual acuity (VA) after 1 year of treatment when compared to other clinical trials with monthly treatment [7]. Moreover, patients with neovascular AMD and initially good visual function (20/40 or better) seem to show comparable treatment results under TER after 2 years [8].

However, there is little data on what to expect in real life when switching from PRN to TER. In our study, we compared functional and anatomical outcomes of patients under long-term therapy with anti-VEGF who have been switched from PRN to TER.

\section{Methods}

Study Design and Patients

This was a 2-year observational, retrospective study at a tertiary referral center.

Patients over 50 years of age with choroidal neovascularization due to AMD with at least 2 years of continuous treatment with aflibercept were included. Within those 2 years, they had at least 1 year of continuous anti-VEGF-therapy using PRN with aflibercept prior to the switch to TER, and they had at least 1 year of continuous treatment under TER. The baseline of analysis was defined as the time point when the switch from PRN to TER occurred.

\section{Regimens}

Pro re nata Regimen. Patients were seen on monthly intervals. However, as this was a retrospective study, some patients had longer intervals due to conflicting schedules. Disease activity was assessed based on OCT. When there was evidence of hemorrhage, subretinal fluid (SRF), or intraretinal fluid (IRF) based on OCT, retreatment was applied; otherwise the patient was scheduled to be seen 4 weeks later.

Treat and Extend Regimen. Patients were seen and treated according to a treat and extend scheme. Extension of intervals were based on signs of activity in OCT. Stability was defined as no IRF or SRF, in which case the retreatment interval was extended by 2 weeks. If there were signs of activity (hemorrhages, IRF, or SRF) the intervals were shortened by 1 week to a minimum of 4 weeks.

Study Procedures

Medical records of patients with CNV secondary to neovascular AMD were reviewed and analyzed retrospectively according to the above-defined criteria. Patients identified from the procedure logs at the Department of Ophthalmology, University Hospital Bern, between November 1, 2012 and July 1, 2015, were reviewed. Patients who underwent intraocular surgery other than intravitreal injections were excluded. Ethics approval (KEK-No. 093/13) to conduct this study was obtained from the local ethics committee, and the study was performed in accordance with ICH-GCP guidelines. The need for written consent from each individual patient was waived because of the retrospective nature of the study.

BCVA and the assessment of IRF and SRF were performed by physicians. Distance BCVA was tested using ETDRS charts, and the BCVA letter score was collected. CRT was measured using Spectralis SD-OCT (Heidelberg Engineering, Heidelberg, Germany; Software version 5.3). Scans were acquired using an established protocol consisting of volume scans. For the volume scan of $20^{\circ} \times$ $20^{\circ}, 49$ frames spaced $121 \mu \mathrm{m}$ and each consisting of 512 A-scans were acquired. The automated real time was set between 6 and 12 to reduce noise. The primary end point of the study was BCVA. Secondary end points were CRT, subretinal fluid and IRF, as well as the numbers of aflibercept injections and visits.

\section{Statistical Analysis}

Data were collected in tables and statistical analysis was performed using the Prism GraphPad ${ }^{\circledR}$ commercial software package (Prism 6; GraphPad Software Inc., La Jolla, CA, USA). Data were analyzed using a paired $t$ test. For all analyses, $p<0.05$ was considered statistically significant. At each timeline, the different categories were tested for the mean value and standard deviation and compared to each other.

\section{Results}

\section{General Data}

Thirty-two patients met our inclusion criteria and were included in this retrospective study ( 24 female and 8 male patients). The mean age was 79.3 years $( \pm 9.5 \mathrm{SD})$. All patients had received previous treatment with antiVEGF agents and were on a long-term treatment course with anti-VEGF agents under PRN, with an average treatment time of $236.1 \pm 95.3$ weeks (or 4.5 years) prior to the switch from PRN to TER.

During the observation time, none of the patients under examination underwent cataract surgery.

\section{Visual Acuity}

During the first year of our study, patients on PRN treatment showed a stable VA (initial BCVA: $64.0 \pm 15.2$; end point BCVA: $64.0 \pm 16.1)$. The mean change in VA was -0.52 letters in the PRN group and -0.65 letters in the TER group compared to baseline (Fig. 1).

\section{$C R T, I R F$, and SRF}

Patients after 1 year of TER treatment had a significant thinner retina (end point: $288 \pm 60 \mu \mathrm{m}$ ) compared to 1 year of treatment using PRN (baseline: $330 \pm 89 \mu \mathrm{m}$; initial CRT: $358 \pm 107 \mu \mathrm{m})(p<0.0001)$. The mean change 


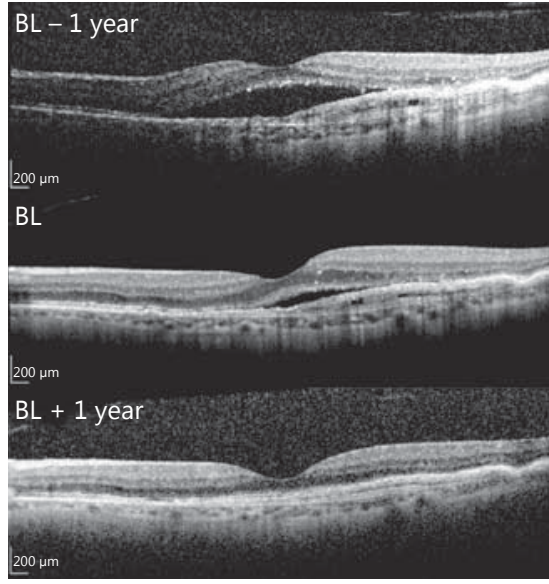

a
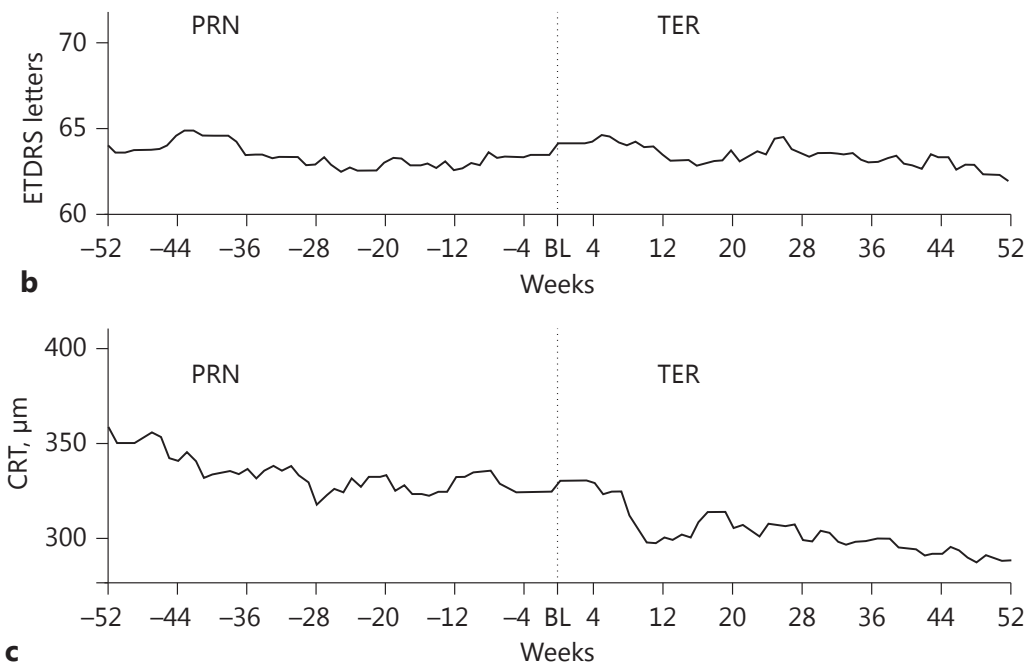

Fig. 1. a Representative OCT images at the beginning of PRN treatment (upper OCT image), at baseline, when the switch of treatment regimens took place (middle OCT image), and after 1 year of TER treatment (lower OCT image). b, c Development of BCVA (upper graph) and CRT (lower graph) over 2 years with a switch of treatment regimen at baseline (BL). The values on the left side of the $\mathrm{BL}$ were recorded within the PRN; whereas on the right side of the BL, TER values are shown. OCT, optical coherence tomography; PRN, pro re nata regimen; TER, treat and extend regimen; CRT, central retinal thickness.

Table 1. OCT characteristics of patients who lost VA of more than 4 letters on the ETDRS chart

\begin{tabular}{lll}
\hline & PRN & TER \\
\hline Patients who lost $\geq$ letters on the ETDRS chart, n (\%) & $8(25)$ & $12(37.5)$ \\
Gender, \% & 62.5 & 66.67 \\
$\quad$ Female & 37.5 & 33.33 \\
$\quad$ Male & 77.25 & 77.0 \\
Mean age, years & 12.5 & 33.3 \\
Increase of IRF, \% & 25 & 16.67 \\
Increase of SRF, \% & 12.5 & 0 \\
Increase of IRF and SRF, \% & 0 & 16.67 \\
Increase of RPE atrophy, \% & 0 & 8.33 \\
Increase of subretinal fibrosis, \% & 12.5 & 0 \\
Opacification of refractive media, \% & 37.5 & 25 \\
No differences/worsening in OCT to determine, \% & & \\
\hline
\end{tabular}

PRN, pro re nata regimen; TER, treat and extend regimen; ETDRS, Early Treatment Diabetic Retinopathy Study; OCT, ocular coherence tomography; RPE, retinal pigment epithelium.

in CRT was $3.02 \mu \mathrm{m}$ in the PRN group and $-25 \mu \mathrm{m}$ in the TER group compared to baseline.

IRF and SRF showed significant changes when switching to TER; both, the percentage of patients with IRF and/or SRF decreased when switching to TER. While $56 \%$ of the patients showed IRF at the beginning of the study (time point: -52 weeks), $49 \%$ had IRF after 1 year of PRN (baseline), and $34 \%$ had IRF after 1 year of TER ( +52 weeks, $p<$ $0.0001)$. At the start of the observation period, $63 \%$ had SRF. After 1 year of treatment with PRN, the percentage of patients having SRF was unchanged and still at $62 \%$, but was reduced to $50 \%$ after 1 year on TER $(p<0.0001)$. 

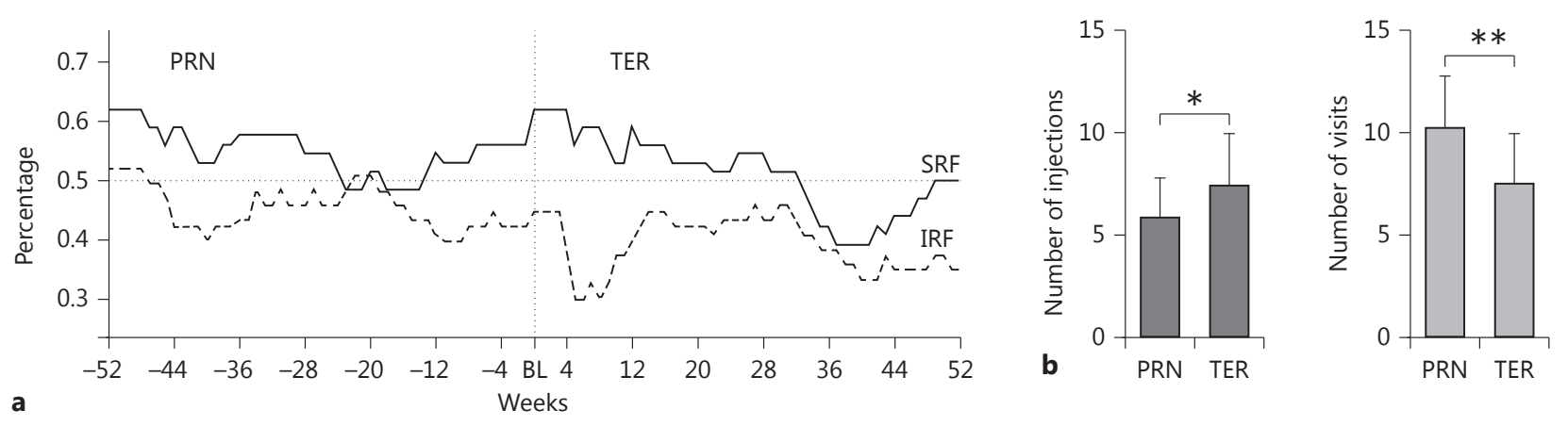

Fig. 2. a The percentage of patients having either SRF or IRF over the course of 2 years with a switch of treatment regimen from PRN to TER at baseline (BL) is shown. b Column graphs depicting the number of injections (black columns) and the number of visits

\section{Subgroups of Patients Who Lost VA}

Next, we investigated those patients who lost VA during the whole observational period $(\geq 4$ letters on the ETDRS reading chart). We identified 20 patients (8 patients during PRN and 12 patients during TER) and recorded the changes seen on OCT and slit-lamp exam of visits where VA dropped (Table 1).

\section{Number of Injections and Visits}

The total number of injections in the PRN timeline group was $5.97 \pm 1.85$ versus $7.5 \pm 2.47$ in the TER timeline group ( $p=0.0002)$. The number of visits was $10.25 \pm$ 2.59 in the PRN period versus $7.5 \pm 2.51$ in the TER pe$\operatorname{riod}(p=<0.0001)$ (Fig. 2b).

\section{Discussion}

The results of our study show that patients being treated with aflibercept on PRN for 1 year and then being switched to TER recorded comparable visual outcomes. However, while the PRN group received fewer injections, the TER group underwent fewer visits and had a significantly decreased CRT after 1 year of treatment.

The change of VA in both regimen groups was similar. A marginal drop of approximately 2 ETDRS letters was seen in the TER period. This may be explained by the natural course of the disease as these patients had already been treated for an average of 2.5 years before being included into this study. Prünte et al. [9] performed a similar study in neovascular AMD patients receiving ranibizumab and evaluated the effect of switching from PRN to (grey columns) in the PRN and the TER group, respectively. SRF, subretinal fluid; IRF, intraretinal fluid; PRN, pro re nata regimen; TER, treat and extend regimen.

TER in a clinical routine setting employing a similar TER protocol. Interestingly, with a similar mean treatment interval of $7.1 \pm 2.3$ weeks, they found a significant improvement of the Snellen BCVA after 1 year of TER $(0.47 \pm 0.22$ to $0.55 \pm 0.23$ ), which is in contrast to our findings. This disagreement may be explained by divergent disease durations of the included patients or by the duration between the last injection on PRN and the first injection on TER.

Although the CRT decreased in the PRN period from $358 \pm 106$ to $330 \pm 89 \mu \mathrm{m}$, CRT further decreased during the TER period. A recent retrospective study analyzing separately PRN and TER cohorts showed a similar decrease in CRT compared to baseline over a period of 3 years [10].

In our study, both the presence of IRF and SRF was significantly different in both treatment regimens. When treatment was switched from PRN to TER, patients showed less fluid in the TER arm (IRF 49 vs. 34\%, SRF 63 vs. 50\%). Fung et al. [11] found comparable results with $50 \%$ SRF and 50\% IRF after 12 months of TER using 0.5 $\mathrm{mg}$ of intravitreal ranibizumab. Nevertheless, aside from our study, there are so far no systematic analyses on IRF and SRF during treatment with TER compared to PRN. However, the goal of TER is to achieve and maintain a "dry" macula by gradually increasing the length of time between injections in the absence of macular fluid $[5,10]$; therefore, our findings underscore this principle.

Similar to the findings of other reports $[10,12]$, patients undergoing antiangiogenic therapy according to the TER dosing scheme received statistically more injections compared to PRN. The mean number of injections our patients received was $7.5 \pm 2.47$ in the TER compared 


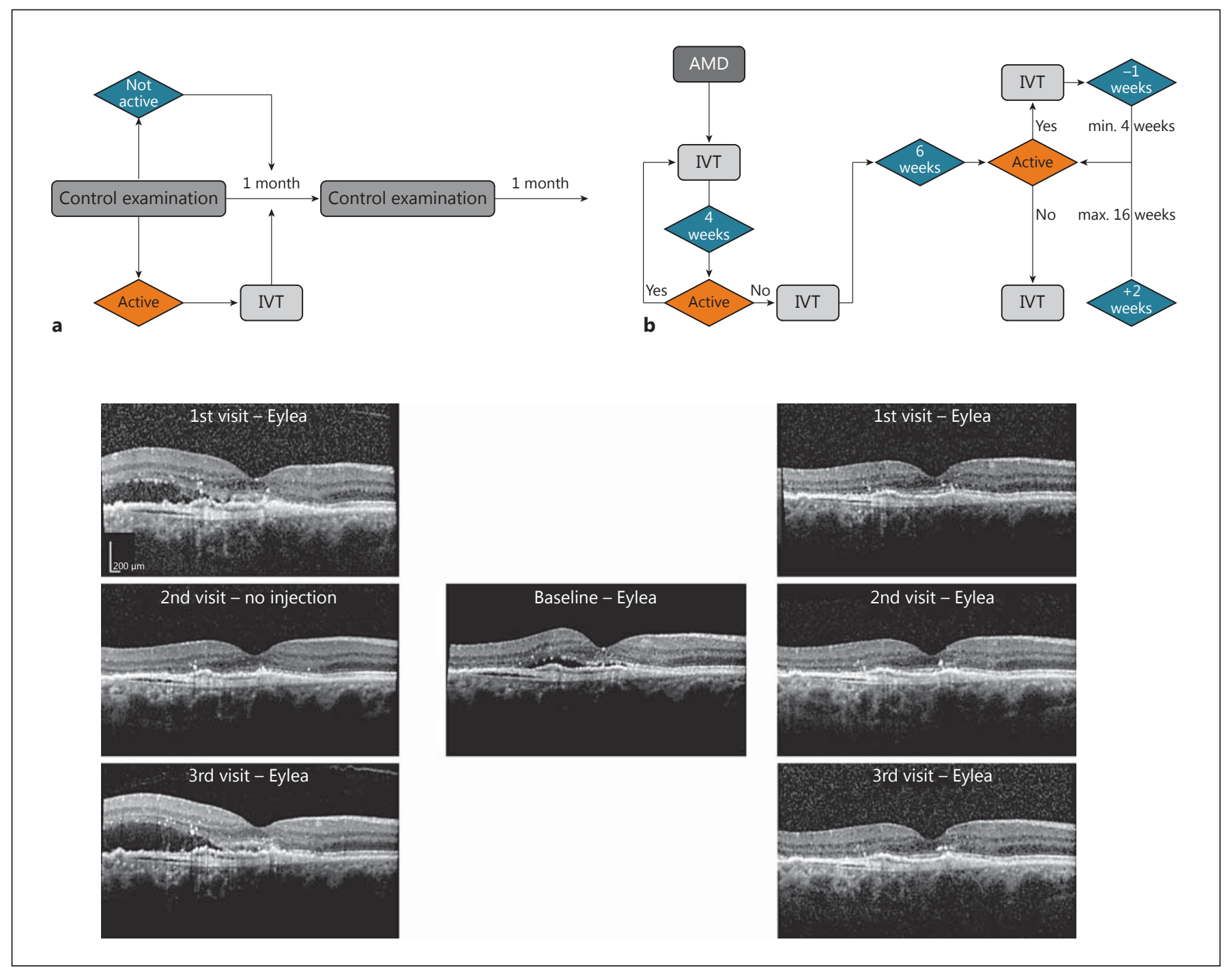

Fig. 3. a Flowchart of PRN treatment (top) and OCT images (bottom) acquired monthly during PRN treatment. An intravitreal injection of Eylea was carried out on every second month. The OCT image in the middle shows the baseline time point when the regimen was switched from PRN to TER. In total, the patient received 7 injections of Eylea and had 11 office visits during the year on PRN

to $5.97 \pm 1.8$ in the PRN. In 1 report, the difference of both groups was recorded as follows: $7.8 \pm 1.3$ injections in the TER versus $5.2 \pm 1.9$ injections in the PRN group [12]. Beside the so far mentioned papers, several other studies were conducted to evaluate the efficacy of TER in neovascular AMD using ranibizumab and bevacizumab $[7,13-$ 16]. Different TER protocols were applied in respective studies but most of them increased the treatment intervals by 2 weeks per visit, unless evidence for disease activity was present. A recent literature review summarized

Results when Switching Treatment

Regimens in AMD Patients treatment. The patient received 4 injections of Lucentis prior to the start of PRN with Eylea. b Flowchart of TER treatment (top) and OCT images on TER (bottom). In total, the patient received 9 injections of Eylea and had the same amount of office visits during that treatment regimen year. OCT, optical coherence tomography; PRN, pro re nata regimen; TER, treat and extend regimen.

the so far conducted TER studies [17]. Based on these studies, a recommended TER algorithm was established, defining disease stability, disease activity, and addressing the extension interval. The consensus panel agreed on an extension interval of 2 weeks if neovascular AMD was stable. In cases of disease activity, the interval should be shortened by 1-2 week. In cases of severe disease activity, a re-induction of monthly treatment should be considered [17]. Our study employed the recommended TER algorithm and therefore gives valid insight into the poten- 
tial benefit when switching from PRN to TER in a routine clinical setting (Fig. 3).

Our study bears several weaknesses, mainly due to its retrospective nature and the relatively low number of patients included. The retrospective design and the "realworld" setting led to visit intervals that were not strictly 4 weekly on PRN, resulting in fewer visits than expected. Generally, the follow-up intervals in TER were better adhered to compared to PRN. Maybe an interval of 4 weeks in the PRN group, as recommended, is not always easy to follow, as it may collide with holidays or other medical appointments in cases of other systemic diseases. Furthermore, because patients were switched from PRN to TER, there is likely to have been more disease progression in the period when TER was applied, which should be considered when assessing the characteristics associated with a significant VA loss under both treatment regimens. Nonetheless, we did not observe any severe recurrence, such as large subretinal bleeding, in between visits within the TER group.
In conclusion, our cohort showed no significant changes in BCVA when switched from PRN to TER. However, the CRT was significantly decreased and there were significantly less patients having IRF and/or SRF. In addition, significantly less visits were observed in the TER period, which may also arise from the fact that TER offers individualized and flexible treatment plans and intervals.

\section{Disclosure Statement}

All authors received nonfinancial support from Heidelberg Engineering $\mathrm{GmBH}$, Germany.

Marion R. Munk received lecturer fees from Novartis (Novartis AG) and travel support from Bayer (Bayer AG). Andreas Ebneter received lecturer fees and travel support from Bayer AG. Sebastian Wolf is consultant for Bayer and Novartis, and grant recipient from Bayer. Martin S. Zinkernagel is consultant for Novartis and Bayer AG, grant recipient from Bayer and stockholder of Novartis Pharmaceuticals.

\section{References}

1 Stewart MW: Individualized treatment of neovascular age-related macular degeneration: what are patients gaining? Or losing? J Clin Med 2015;4:1079-1101.

2 Chakravarthy U, Harding SP, Rogers CA, Downes SM, Lotery AJ, Culliford LA, Reeves BC; IVAN study investigators: Alternative treatments to inhibit VEGF in age-related choroidal neovascularisation: 2-year findings of the IVAN randomised controlled trial. Lancet 2013;382:1258-1267.

3 Comparison of Age-related Macular Degeneration Treatments Trials (CATT) Research Group; Martin DF, Maguire MG, Fine SL, Ying GS, Jaffe GJ, Grunwald JE, Toth C, Redford M, Ferris FL 3rd: Ranibizumab and bevacizumab for treatment of neovascular agerelated macular degeneration: two-year results. Ophthalmology 2012;119:1388-1398.

4 Lalwani GA, Rosenfeld PJ, Fung AE, Dubovy SR, Michels S, Feuer W, Davis JL, Flynn HW Jr, Esquiabro M: A variable-dosing regimen with intravitreal ranibizumab for neovascular age-related macular degeneration: year 2 of the PrONTO Study. Am J Ophthalmol 2009; 148:43-58.e1.

5 Spaide R: Ranibizumab according to need: a treatment for age-related macular degeneration. Am J Ophthalmol 2007;143:679-680.

6 Spaide RF: The as-needed treatment strategy for choroidal neovascularization: a feedbackbased treatment system. Am J Ophthalmol 2009;148:1-3.
7 Berg K, Pedersen TR, Sandvik L, Bragadottir $\mathrm{R}$ : Comparison of ranibizumab and bevacizumab for neovascular age-related macular degeneration according to LUCAS treat-andextend protocol. Ophthalmology 2015;122: 146-152.

8 Rahimy E, Rayess N, Ho AC, Regillo CD: Treatment outcomes for neovascular age-related macular degeneration patients with initial vision better than $20 / 40$ using a treat-andextend regimen. Retina 2016;36:875-880.

9 Prünte C, Hatz K: Switch over from PRN to treat and extend in exudative AMD: 1-year results from a clinical series. Macula Society 37th Annual Meeting 2014, Key Largo Florida, February 20, 2014.

10 Calvo P, Wang Y, Ferreras A, Lam W-C, Devenyi $R$, Brent $M H$ : Treat and extend versus treat and observe regimens in wet age-related macular degeneration patients treated with ranibizumab: 3-year surveillance period. J Clin Exp Ophthalmol 2014;5:324.

11 Fung AT, Kumar N, Vance SK, Slakter JS, Klancnik JM, Spaide RS, Freund KB: Pilot study to evaluate the role of high-dose ranibizumab $2.0 \mathrm{mg}$ in the management of neovascular age-related macular degeneration in patients with persistent/recurrent macular fluid $<30$ days following treatment with intravitreal anti-vegf therapy (the last study). Eye 2012;26:1181-1187.
12 Oubraham H, Cohen SY, Samimi S, Marotte D, Bouzaher I, Bonicel P, Fajnkuchen F, Tadayoni R: Inject and extend dosing versus dosing as needed: a comparative retrospective study of ranibizumab in exudative age-related macular degeneration. Retina 2011;31:26-30.

13 Abedi F, Wickremasinghe S, Islam AF, Inglis $\mathrm{KM}$, Guymer RH: Anti-VEGF treatment in neovascular age-related macular degeneration: a treat-and-extend protocol over 2 years. Retina 2014;34:1531-1538.

14 Engelbert M, Zweifel SA, Freund KB: "Treat and extend" dosing of intravitreal antivascular endothelial growth factor therapy for type 3 neovascularization/retinal angiomatous proliferation. Retina 2009;29:1424-1431.

15 Toalster N, Russell M, Ng P: A 12-month prospective trial of inject and extend regimen for ranibizumab treatment of age-related macular degeneration. Retina 2013;33:1351-1358.

16 Wykoff CC, Croft DE, Brown DM, Wang R, Payne JF, Clark L, Abdelfattah NS, Sadda SR; TREX-AMD Study Group: Prospective trial of treat-and-extend versus monthly dosing for neovascular age-related macular degeneration: TREX-AMD 1-year results. Ophthalmology 2015;122:2514-2522.

17 Freund KB, Korobelnik JF, Devenyi R, Framme C, Galic J, Herbert E, Hoerauf $\mathrm{H}$, Lanzetta P, Michels S, Mitchell P, Mones J, Regillo C, Tadayoni R, Talks J, Wolf S: Treatand-extend regimens with anti-VEGF agents in retinal diseases: a literature review and consensus recommendations. Retina 2015;35: 1489-1506. 